\title{
Dysgeusia, CTCAE
}

National Cancer Institute

\section{Source}

National Cancer Institute. Dysgeusia, CT CAE. NCI Thesaurus. Code C143422.

A disorder characterized by abnormal sensual experience with the taste of foodstuffs; it can be related to a decrease in the sense of smell. 